\title{
Synthesis of monodentate ferrocenylphosphines and their application to the palladium catalysed Suzuki reaction of aryl chlorides
}

Tom E. Pickett, Francesc X. Roca and Christopher J. Richards

\section{Supplementary Material}

Table of Contents

Page S2. Table S1. Crystal data and structure refinement for 5.

Page S3. Table S2. Atomic coordinates ( $\quad 10^{4}$ ) and equivalent isotropic displacement parameters $\left(\AA^{2} \times 10^{3}\right)$ for 5 .

Page S4. Table S3. Bond lengths $[\AA ̊]$ and angles $\left[{ }^{\circ}\right]$ for $\mathbf{5}$.

Page S13. Table S4. Anisotropic displacement parameters ffor 5.

Page S15. ${ }^{1} \mathrm{H}$ NMR of 2-methyl-1-diphenylphosphinoferrocene 11.

Page S16. ${ }^{31} \mathrm{P}$ NMR of 2-methyl-1-diphenylphosphinoferrocene 11.

Page S17. ${ }^{1} \mathrm{H}$ NMR of 2-methyl-1-dicyclohexylphosphinoferrocene 12.

Page S18. ${ }^{31} \mathrm{P}$ NMR of 2-methyl-1-dicyclohexylphosphinoferrocene12.

Page S19. ${ }^{1} \mathrm{H}$ NMR of 2-methyl-1-di-tert-butylphosphinoferrocene 13.

Page S20. ${ }^{31} \mathrm{P}$ NMR of 2-methyl-1-di-tert-butylphosphinoferrocene 13. 
Table S1. Crystal data and structure refinement for $\mathbf{5}$.

Identification code

Empirical formula

Formula weight

Temperature

Wavelength

Crystal system

Space group

Unit cell dimensions

Volume

$\mathrm{Z}$

Density (calculated)

Absorption coefficient

$\mathrm{F}(000)$

Crystal size

Theta range for data collection

Index ranges

Reflections collected

Independent reflections

Completeness to theta $=25.01^{\circ}$

Max. and min. transmission

Refinement method

Data / restraints / parameters

Goodness-of-fit on $\mathrm{F}^{2}$

Final R indices [I $>2 \operatorname{sigma}(\mathrm{I})]$

$\mathrm{R}$ indices (all data)

Absolute structure parameter

Largest diff. peak and hole
XR-72

$\mathrm{C}_{33} \mathrm{H}_{33} \mathrm{Fe}_{3} \mathrm{P}$

628.11

160(2) K

$0.71073 \AA$

Trigonal

R3

$\mathrm{a}=18.723(4) \AA$

$\alpha=90^{\circ}$.

$\mathrm{b}=18.723(4) \AA$

$\beta=90^{\circ}$.

$\mathrm{c}=12.991(6) \AA$

$\gamma=120^{\circ}$.
6

$1.587 \mathrm{Mg} / \mathrm{m}^{3}$

$1.716 \mathrm{~mm}^{-1}$

1944

$0.40 \times 0.40 \times 0.20 \mathrm{~mm}^{3}$

2.01 to $25.01^{\circ}$.

$-22<=\mathrm{h}<=13,-13<=\mathrm{k}<=22,-14<=\mathrm{l}<=15$

4953

$2969[\mathrm{R}(\mathrm{int})=0.0424]$

$99.7 \%$

0.7253 and 0.5469

Full-matrix least-squares on $\mathrm{F}^{2}$

2969 / 1 / 311

1.031

$\mathrm{R} 1=0.0231, \mathrm{wR} 2=0.0612$

$\mathrm{R} 1=0.0234, \mathrm{wR} 2=0.0615$

$0.015(12)$

0.304 and -0.505 e. $\AA^{-3}$ 
Table S2. Atomic coordinates ( x 10 $)$ and equivalent isotropic displacement parameters $\left(\AA^{2} \times 10^{3}\right)$ . U(eq) is defined as one third of the trace of the orthogonalized $\mathrm{U}^{\mathrm{ij}}$ tensor.

\begin{tabular}{|c|c|c|c|c|}
\hline & $\mathrm{X}$ & $\mathrm{y}$ & $\mathrm{z}$ & $\mathrm{U}(\mathrm{eq})$ \\
\hline $\mathrm{C}(1)$ & $7230(1)$ & $4340(2)$ & $-136(2)$ & $14(1)$ \\
\hline $\mathrm{C}(2)$ & $7280(2)$ & $4532(1)$ & $932(2)$ & $16(1)$ \\
\hline $\mathrm{C}(3)$ & $7835(2)$ & $5384(2)$ & $1065(2)$ & $20(1)$ \\
\hline $\mathrm{C}(4)$ & $8132(2)$ & $5736(2)$ & $75(2)$ & $21(1)$ \\
\hline$C(5)$ & $7756(1)$ & $5096(1)$ & $-676(2)$ & $16(1)$ \\
\hline$C(6)$ & $5674(2)$ & $4772(2)$ & $534(3)$ & $28(1)$ \\
\hline$C(7)$ & $6171(2)$ & $5592(2)$ & $869(2)$ & $29(1)$ \\
\hline $\mathrm{C}(8)$ & $6562(2)$ & $6092(2)$ & $-5(2)$ & $24(1)$ \\
\hline $\mathrm{C}(9)$ & $6298(2)$ & $5580(2)$ & $-890(2)$ & $23(1)$ \\
\hline $\mathrm{C}(10)$ & $5749(2)$ & $4763(2)$ & $-555(2)$ & $26(1)$ \\
\hline $\mathrm{C}(11)$ & $3748(2)$ & $4309(2)$ & $-843(2)$ & $23(1)$ \\
\hline$C(12)$ & $3354(2)$ & $3843(2)$ & $-1732(2)$ & $25(1)$ \\
\hline$C(13)$ & $3637(2)$ & $4378(2)$ & $-2594(2)$ & $29(1)$ \\
\hline $\mathrm{C}(14)$ & $4219(2)$ & $5178(2)$ & $-2231(3)$ & $30(1)$ \\
\hline$C(15)$ & $4283(2)$ & $5137(2)$ & $-1149(2)$ & $27(1)$ \\
\hline$C(16)$ & $2132(2)$ & $4578(2)$ & $-608(2)$ & $20(1)$ \\
\hline$C(17)$ & $1835(2)$ & $4260(2)$ & $-1603(2)$ & $21(1)$ \\
\hline $\mathrm{C}(18)$ & $2227(1)$ & 4919(1) & $-2328(2)$ & $17(1)$ \\
\hline$C(19)$ & $2764(1)$ & $5659(1)$ & $-1767(2)$ & $15(1)$ \\
\hline $\mathrm{C}(20)$ & $2705(2)$ & $5433(2)$ & $-699(2)$ & $18(1)$ \\
\hline$C(21)$ & $2109(2)$ & $4842(2)$ & $-3471(2)$ & $25(1)$ \\
\hline$C(22)$ & $7895(2)$ & $5220(2)$ & $-1811(2)$ & $24(1)$ \\
\hline $\mathrm{Fe}(1)$ & $6872(1)$ & 5191(1) & 130(1) & $15(1)$ \\
\hline $\mathrm{Fe}(2)$ & 3092(1) & $4776(1)$ & $-1555(1)$ & $16(1)$ \\
\hline $\mathrm{P}(3)$ & 6667 & 3333 & $-771(1)$ & $12(1)$ \\
\hline $\mathrm{P}(4)$ & 3333 & 6667 & $-2377(1)$ & $13(1)$ \\
\hline
\end{tabular}


Table S3. Bond lengths $[\AA]$ and angles $\left[^{\circ}\right]$.

\begin{tabular}{ll}
\hline $\mathrm{C}(1)-\mathrm{C}(2)$ & $1.425(3)$ \\
$\mathrm{C}(1)-\mathrm{C}(5)$ & $1.440(3)$ \\
$\mathrm{C}(1)-\mathrm{P}(3)$ & $1.831(2)$ \\
$\mathrm{C}(1)-\mathrm{Fe}(1)$ & $2.044(2)$ \\
$\mathrm{C}(2)-\mathrm{C}(3)$ & $1.413(3)$ \\
$\mathrm{C}(2)-\mathrm{Fe}(1)$ & $2.034(2)$ \\
$\mathrm{C}(2)-\mathrm{H}(2)$ & $0.90(3)$ \\
$\mathrm{C}(3)-\mathrm{C}(4)$ & $1.425(4)$ \\
$\mathrm{C}(3)-\mathrm{Fe}(1)$ & $2.051(2)$ \\
$\mathrm{C}(3)-\mathrm{H}(3)$ & $0.89(4)$ \\
$\mathrm{C}(4)-\mathrm{C}(5)$ & $1.429(4)$ \\
$\mathrm{C}(4)-\mathrm{Fe}(1)$ & $2.050(3)$ \\
$\mathrm{C}(4)-\mathrm{H}(4)$ & $0.96(3)$ \\
$\mathrm{C}(5)-\mathrm{C}(22)$ & $1.495(4)$ \\
$\mathrm{C}(5)-\mathrm{Fe}(1)$ & $2.040(2)$ \\
$\mathrm{C}(6)-\mathrm{C}(7)$ & $1.409(5)$ \\
$\mathrm{C}(6)-\mathrm{C}(10)$ & $1.422(5)$ \\
$\mathrm{C}(6)-\mathrm{Fe}(1)$ & $2.040(3)$ \\
$\mathrm{C}(6)-\mathrm{H}(6)$ & $1.02(4)$ \\
$\mathrm{C}(7)-\mathrm{C}(8)$ & $1.420(4)$ \\
$\mathrm{C}(7)-\mathrm{Fe}(1)$ & $2.048(3)$ \\
$\mathrm{C}(7)-\mathrm{H}(7)$ & $0.94(4)$ \\
$\mathrm{C}(8)-\mathrm{C}(9)$ & $1.419(4)$ \\
$\mathrm{C}(8)-\mathrm{Fe}(1)$ & $2.048(3)$ \\
$\mathrm{C}(8)-\mathrm{H}(8)$ & $0.91(3)$ \\
$\mathrm{C}(9)-\mathrm{C}(10)$ & $1.418(4)$ \\
$\mathrm{C}(9)-\mathrm{Fe}(1)$ & $2.055(3)$ \\
$\mathrm{C}(9)-\mathrm{H}(9)$ & $0.95(3)$ \\
$\mathrm{C}(10)-\mathrm{Fe}(1)$ & $2.042(3)$ \\
$\mathrm{C}(10)-\mathrm{H}(10)$ & $0.94(3)$ \\
$\mathrm{C}(11)-\mathrm{C}(12)$ & $1.412(4)$ \\
$\mathrm{C}(11)-\mathrm{C}(15)$ & $1.418(4)$ \\
$\mathrm{C}(11)-\mathrm{Fe}(2)$ & $2.049(3)$ \\
$\mathrm{C}(11)-\mathrm{H}(11)$ & $0.92(4)$ \\
$\mathrm{C}(12)-\mathrm{C}(13)$ & \\
& \\
&
\end{tabular}




$\begin{array}{ll}\mathrm{C}(12)-\mathrm{Fe}(2) & 2.049(3) \\ \mathrm{C}(12)-\mathrm{H}(12) & 0.99(3) \\ \mathrm{C}(13)-\mathrm{C}(14) & 1.422(4) \\ \mathrm{C}(13)-\mathrm{Fe}(2) & 2.044(3) \\ \mathrm{C}(13)-\mathrm{H}(13) & 0.92(4) \\ \mathrm{C}(14)-\mathrm{C}(15) & 1.416(5) \\ \mathrm{C}(14)-\mathrm{Fe}(2) & 2.050(3) \\ \mathrm{C}(14)-\mathrm{H}(14) & 0.90(4) \\ \mathrm{C}(15)-\mathrm{Fe}(2) & 2.050(3) \\ \mathrm{C}(15)-\mathrm{H}(15) & 0.91(4) \\ \mathrm{C}(16)-\mathrm{C}(17) & 1.416(4) \\ \mathrm{C}(16)-\mathrm{C}(20) & 1.417(4) \\ \mathrm{C}(16)-\mathrm{Fe}(2) & 2.052(2) \\ \mathrm{C}(16)-\mathrm{H}(16) & 0.92(4) \\ \mathrm{C}(17)-\mathrm{C}(18) & 1.429(4) \\ \mathrm{C}(17)-\mathrm{Fe}(2) & 2.050(3) \\ \mathrm{C}(17)-\mathrm{H}(17) & 0.96(3) \\ \mathrm{C}(18)-\mathrm{C}(19) & 1.438(3) \\ \mathrm{C}(18)-\mathrm{C}(21) & 1.498(3) \\ \mathrm{C}(18)-\mathrm{Fe}(2) & 2.034(2) \\ \mathrm{C}(19)-\mathrm{C}(20) & 1.438(3) \\ \mathrm{C}(19)-\mathrm{P}(4) & 1.820(2) \\ \mathrm{C}(19)-\mathrm{Fe}(2) & 2.052(2) \\ \mathrm{C}(20)-\mathrm{Fe}(2) & 2.041(2) \\ \mathrm{C}(20)-\mathrm{H}(20) & 0.96(3) \\ \mathrm{C}(21)-\mathrm{H}(21 \mathrm{~A}) & 1.00(4) \\ \mathrm{C}(21)-\mathrm{H}(21 \mathrm{~B}) & 0.98(4) \\ \mathrm{C}(21)-\mathrm{H}(21 \mathrm{C}) & 0.88(4) \\ \mathrm{C}(22)-\mathrm{H}(22 \mathrm{~A}) & 0.96(4) \\ \mathrm{C}(22)-\mathrm{H}(22 \mathrm{~B}) & 0.90(4) \\ \mathrm{C}(22)-\mathrm{H}(22 \mathrm{C}) & 0.88(4) \\ \mathrm{P}(3)-\mathrm{C}(1) \# 1 & 1.831(2) \\ \mathrm{P}(3)-\mathrm{C}(1) \# 2 & 1.831(2) \\ \mathrm{P}(4)-\mathrm{C}(19) \# 3 & 1.820(2) \\ \mathrm{P}(4)-\mathrm{C}(19) \# 4 & 1.820(2) \\ \mathrm{C}(2)-\mathrm{C}(1)-\mathrm{C}(5) & \\ & 107.4(2)\end{array}$




$\begin{array}{lc}\mathrm{C}(2)-\mathrm{C}(1)-\mathrm{P}(3) & 129.00(18) \\ \mathrm{C}(5)-\mathrm{C}(1)-\mathrm{P}(3) & 123.47(18) \\ \mathrm{C}(2)-\mathrm{C}(1)-\mathrm{Fe}(1) & 69.19(13) \\ \mathrm{C}(5)-\mathrm{C}(1)-\mathrm{Fe}(1) & 69.22(13) \\ \mathrm{P}(3)-\mathrm{C}(1)-\mathrm{Fe}(1) & 129.31(12) \\ \mathrm{C}(3)-\mathrm{C}(2)-\mathrm{C}(1) & 108.8(2) \\ \mathrm{C}(3)-\mathrm{C}(2)-\mathrm{Fe}(1) & 70.41(14) \\ \mathrm{C}(1)-\mathrm{C}(2)-\mathrm{Fe}(1) & 69.92(14) \\ \mathrm{C}(3)-\mathrm{C}(2)-\mathrm{H}(2) & 126.3(18) \\ \mathrm{C}(1)-\mathrm{C}(2)-\mathrm{H}(2) & 124.5(18) \\ \mathrm{Fe}(1)-\mathrm{C}(2)-\mathrm{H}(2) & 120.0(16) \\ \mathrm{C}(2)-\mathrm{C}(3)-\mathrm{C}(4) & 107.9(2) \\ \mathrm{C}(2)-\mathrm{C}(3)-\mathrm{Fe}(1) & 69.11(13) \\ \mathrm{C}(4)-\mathrm{C}(3)-\mathrm{Fe}(1) & 69.63(15) \\ \mathrm{C}(2)-\mathrm{C}(3)-\mathrm{H}(3) & 122(2) \\ \mathrm{C}(4)-\mathrm{C}(3)-\mathrm{H}(3) & 130(2) \\ \mathrm{Fe}(1)-\mathrm{C}(3)-\mathrm{H}(3) & 125(2) \\ \mathrm{C}(3)-\mathrm{C}(4)-\mathrm{C}(5) & 108.3(2) \\ \mathrm{C}(3)-\mathrm{C}(4)-\mathrm{Fe}(1) & 69.70(15) \\ \mathrm{C}(5)-\mathrm{C}(4)-\mathrm{Fe}(1) & 69.20(13) \\ \mathrm{C}(3)-\mathrm{C}(4)-\mathrm{H}(4) & 122.0(19) \\ \mathrm{C}(5)-\mathrm{C}(4)-\mathrm{H}(4) & 129.6(19) \\ \mathrm{Fe}(1)-\mathrm{C}(4)-\mathrm{H}(4) & 124.7(18) \\ \mathrm{C}(4)-\mathrm{C}(5)-\mathrm{C}(1) & 107.4(2) \\ \mathrm{C}(4)-\mathrm{C}(5)-\mathrm{C}(22) & 124.7(2) \\ \mathrm{C}(1)-\mathrm{C}(5)-\mathrm{C}(22) & 127.8(2) \\ \mathrm{C}(4)-\mathrm{C}(5)-\mathrm{Fe}(1) & 69.92(14) \\ \mathrm{C}(1)-\mathrm{C}(5)-\mathrm{Fe}(1) & 69.48(13) \\ \mathrm{C}(22)-\mathrm{C}(5)-\mathrm{Fe}(1) & 125.37(18) \\ \mathrm{C}(7)-\mathrm{C}(6)-\mathrm{C}(10) & 107.7(2) \\ \mathrm{C}(7)-\mathrm{C}(6)-\mathrm{Fe}(1) & 70.13(16) \\ \mathrm{C}(10)-\mathrm{C}(6)-\mathrm{Fe}(1) & 69.69(15) \\ \mathrm{C}(7)-\mathrm{C}(6)-\mathrm{H}(6) & 124(2) \\ \mathrm{C}(10)-\mathrm{C}(6)-\mathrm{H}(6) & 128(2) \\ \mathrm{Fe}(1)-\mathrm{C}(6)-\mathrm{H}(6) & 125(2) \\ \mathrm{C}(6)-\mathrm{C}(7)-\mathrm{C}(8) & 108.2(3) \\ \mathrm{C}(6)-\mathrm{C}(7)-\mathrm{Fe}(1) & 69.56(15) \\ & \end{array}$




$\begin{array}{ll}\mathrm{C}(8)-\mathrm{C}(7)-\mathrm{Fe}(1) & 69.72(16) \\ \mathrm{C}(6)-\mathrm{C}(7)-\mathrm{H}(7) & 122(2) \\ \mathrm{C}(8)-\mathrm{C}(7)-\mathrm{H}(7) & 130(2) \\ \mathrm{Fe}(1)-\mathrm{C}(7)-\mathrm{H}(7) & 126(2) \\ \mathrm{C}(9)-\mathrm{C}(8)-\mathrm{C}(7) & 108.2(2) \\ \mathrm{C}(9)-\mathrm{C}(8)-\mathrm{Fe}(1) & 70.03(15) \\ \mathrm{C}(7)-\mathrm{C}(8)-\mathrm{Fe}(1) & 69.72(15) \\ \mathrm{C}(9)-\mathrm{C}(8)-\mathrm{H}(8) & 128(2) \\ \mathrm{C}(7)-\mathrm{C}(8)-\mathrm{H}(8) & 124(2) \\ \mathrm{Fe}(1)-\mathrm{C}(8)-\mathrm{H}(8) & 123.6(19) \\ \mathrm{C}(10)-\mathrm{C}(9)-\mathrm{C}(8) & 107.4(2) \\ \mathrm{C}(10)-\mathrm{C}(9)-\mathrm{Fe}(1) & 69.27(15) \\ \mathrm{C}(8)-\mathrm{C}(9)-\mathrm{Fe}(1) & 69.51(15) \\ \mathrm{C}(10)-\mathrm{C}(9)-\mathrm{H}(9) & 127.0(16) \\ \mathrm{C}(8)-\mathrm{C}(9)-\mathrm{H}(9) & 125.5(16) \\ \mathrm{Fe}(1)-\mathrm{C}(9)-\mathrm{H}(9) & 123.9(16) \\ \mathrm{C}(9)-\mathrm{C}(10)-\mathrm{C}(6) & 108.4(2) \\ \mathrm{C}(9)-\mathrm{C}(10)-\mathrm{Fe}(1) & 70.22(15) \\ \mathrm{C}(6)-\mathrm{C}(10)-\mathrm{Fe}(1) & 69.55(16) \\ \mathrm{C}(9)-\mathrm{C}(10)-\mathrm{H}(10) & 129.7(17) \\ \mathrm{C}(6)-\mathrm{C}(10)-\mathrm{H}(10) & 121.9(17) \\ \mathrm{Fe}(1)-\mathrm{C}(10)-\mathrm{H}(10) & 125.4(17) \\ \mathrm{C}(12)-\mathrm{C}(11)-\mathrm{C}(15) & 108.2(2) \\ \mathrm{C}(12)-\mathrm{C}(11)-\mathrm{Fe}(2) & 69.83(15) \\ \mathrm{C}(15)-\mathrm{C}(11)-\mathrm{Fe}(2) & 69.78(15) \\ \mathrm{C}(12)-\mathrm{C}(11)-\mathrm{H}(11) & 124(2) \\ \mathrm{C}(15)-\mathrm{C}(11)-\mathrm{H}(11) & 127(2) \\ \mathrm{Fe}(2)-\mathrm{C}(11)-\mathrm{H}(11) & 126(2) \\ \mathrm{C}(11)-\mathrm{C}(12)-\mathrm{C}(13) & 108.3(2) \\ \mathrm{C}(11)-\mathrm{C}(12)-\mathrm{Fe}(2) & 69.87(15) \\ \mathrm{C}(13)-\mathrm{C}(12)-\mathrm{Fe}(2) & 69.55(14) \\ \mathrm{C}(11)-\mathrm{C}(12)-\mathrm{H}(12) & 127(2) \\ \mathrm{C}(13)-\mathrm{C}(12)-\mathrm{H}(12) & 124(2) \\ \mathrm{Fe}(2)-\mathrm{C}(12)-\mathrm{H}(12) & 123.3(19) \\ \mathrm{C}(12)-\mathrm{C}(13)-\mathrm{C}(14) & 107.5(3) \\ \mathrm{C}(12)-\mathrm{C}(13)-\mathrm{Fe}(2) & 69.92(15) \\ \mathrm{C}(14)-\mathrm{C}(13)-\mathrm{Fe}(2) & 69.90(15) \\ & \end{array}$




$\begin{array}{ll}\mathrm{C}(12)-\mathrm{C}(13)-\mathrm{H}(13) & 127(2) \\ \mathrm{C}(14)-\mathrm{C}(13)-\mathrm{H}(13) & 126(2) \\ \mathrm{Fe}(2)-\mathrm{C}(13)-\mathrm{H}(13) & 123(2) \\ \mathrm{C}(15)-\mathrm{C}(14)-\mathrm{C}(13) & 108.3(3) \\ \mathrm{C}(15)-\mathrm{C}(14)-\mathrm{Fe}(2) & 69.79(17) \\ \mathrm{C}(13)-\mathrm{C}(14)-\mathrm{Fe}(2) & 69.45(16) \\ \mathrm{C}(15)-\mathrm{C}(14)-\mathrm{H}(14) & 125(2) \\ \mathrm{C}(13)-\mathrm{C}(14)-\mathrm{H}(14) & 126(2) \\ \mathrm{Fe}(2)-\mathrm{C}(14)-\mathrm{H}(14) & 128(2) \\ \mathrm{C}(14)-\mathrm{C}(15)-\mathrm{C}(11) & 107.7(3) \\ \mathrm{C}(14)-\mathrm{C}(15)-\mathrm{Fe}(2) & 69.79(17) \\ \mathrm{C}(11)-\mathrm{C}(15)-\mathrm{Fe}(2) & 69.74(15) \\ \mathrm{C}(14)-\mathrm{C}(15)-\mathrm{H}(15) & 127(2) \\ \mathrm{C}(11)-\mathrm{C}(15)-\mathrm{H}(15) & 125(2) \\ \mathrm{Fe}(2)-\mathrm{C}(15)-\mathrm{H}(15) & 128(2) \\ \mathrm{C}(17)-\mathrm{C}(16)-\mathrm{C}(20) & 108.4(2) \\ \mathrm{C}(17)-\mathrm{C}(16)-\mathrm{Fe}(2) & 69.74(15) \\ \mathrm{C}(20)-\mathrm{C}(16)-\mathrm{Fe}(2) & 69.32(14) \\ \mathrm{C}(17)-\mathrm{C}(16)-\mathrm{H}(16) & 121(2) \\ \mathrm{C}(20)-\mathrm{C}(16)-\mathrm{H}(16) & 130(2) \\ \mathrm{Fe}(2)-\mathrm{C}(16)-\mathrm{H}(16) & 123(2) \\ \mathrm{C}(16)-\mathrm{C}(17)-\mathrm{C}(18) & 108.3(2) \\ \mathrm{C}(16)-\mathrm{C}(17)-\mathrm{Fe}(2) & 69.88(14) \\ \mathrm{C}(18)-\mathrm{C}(17)-\mathrm{Fe}(2) & 68.89(14) \\ \mathrm{C}(16)-\mathrm{C}(17)-\mathrm{H}(17) & 128(2) \\ \mathrm{C}(18)-\mathrm{C}(17)-\mathrm{H}(17) & 124(2) \\ \mathrm{Fe}(2)-\mathrm{C}(17)-\mathrm{H}(17) & 127(2) \\ \mathrm{C}(17)-\mathrm{C}(18)-\mathrm{C}(19) & 108.0(2) \\ \mathrm{C}(17)-\mathrm{C}(18)-\mathrm{C}(21) & 125.6(2) \\ \mathrm{C}(19)-\mathrm{C}(18)-\mathrm{C}(21) & 126.4(2) \\ \mathrm{C}(17)-\mathrm{C}(18)-\mathrm{Fe}(2) & 70.15(14) \\ \mathrm{C}(19)-\mathrm{C}(18)-\mathrm{Fe}(2) & 70.06(13) \\ \mathrm{C}(21)-\mathrm{C}(18)-\mathrm{Fe}(2) & 124.43(18) \\ \mathrm{C}(18)-\mathrm{C}(19)-\mathrm{C}(20) & 106.9(2) \\ \mathrm{C}(18)-\mathrm{C}(19)-\mathrm{P}(4) & 122.89(18) \\ \mathrm{C}(20)-\mathrm{C}(19)-\mathrm{P}(4) & 130.11(19) \\ \mathrm{C}(18)-\mathrm{C}(19)-\mathrm{Fe}(2) & 68.72(13) \\ & \end{array}$




$\begin{array}{lc}\mathrm{C}(20)-\mathrm{C}(19)-\mathrm{Fe}(2) & 69.03(13) \\ \mathrm{P}(4)-\mathrm{C}(19)-\mathrm{Fe}(2) & 129.61(12) \\ \mathrm{C}(16)-\mathrm{C}(20)-\mathrm{C}(19) & 108.4(2) \\ \mathrm{C}(16)-\mathrm{C}(20)-\mathrm{Fe}(2) & 70.17(14) \\ \mathrm{C}(19)-\mathrm{C}(20)-\mathrm{Fe}(2) & 69.82(14) \\ \mathrm{C}(16)-\mathrm{C}(20)-\mathrm{H}(20) & 125.1(18) \\ \mathrm{C}(19)-\mathrm{C}(20)-\mathrm{H}(20) & 126.4(18) \\ \mathrm{Fe}(2)-\mathrm{C}(20)-\mathrm{H}(20) & 125.1(18) \\ \mathrm{C}(18)-\mathrm{C}(21)-\mathrm{H}(21 \mathrm{~A}) & 116(2) \\ \mathrm{C}(18)-\mathrm{C}(21)-\mathrm{H}(21 \mathrm{~B}) & 113(2) \\ \mathrm{H}(21 \mathrm{~A})-\mathrm{C}(21)-\mathrm{H}(21 \mathrm{~B}) & 105(3) \\ \mathrm{C}(18)-\mathrm{C}(21)-\mathrm{H}(21 \mathrm{C}) & 104(3) \\ \mathrm{H}(21 \mathrm{~A})-\mathrm{C}(21)-\mathrm{H}(21 \mathrm{C}) & 115(3) \\ \mathrm{H}(21 \mathrm{~B})-\mathrm{C}(21)-\mathrm{H}(21 \mathrm{C}) & 104(3) \\ \mathrm{C}(5)-\mathrm{C}(22)-\mathrm{H}(22 \mathrm{~A}) & 107(3) \\ \mathrm{C}(5)-\mathrm{C}(22)-\mathrm{H}(22 \mathrm{~B}) & 113(2) \\ \mathrm{H}(22 \mathrm{~A})-\mathrm{C}(22)-\mathrm{H}(22 \mathrm{~B}) & 103(3) \\ \mathrm{C}(5)-\mathrm{C}(22)-\mathrm{H}(22 \mathrm{C}) & 112(3) \\ \mathrm{H}(22 \mathrm{~A})-\mathrm{C}(22)-\mathrm{H}(22 \mathrm{C}) & 111(3) \\ \mathrm{H}(22 \mathrm{~B})-\mathrm{C}(22)-\mathrm{H}(22 \mathrm{C}) & 111(3) \\ \mathrm{C}(2)-\mathrm{Fe}(1)-\mathrm{C}(6) & 108.06(11) \\ \mathrm{C}(2)-\mathrm{Fe}(1)-\mathrm{C}(5) & 69.07(10) \\ \mathrm{C}(6)-\mathrm{Fe}(1)-\mathrm{C}(5) & 151.97(11) \\ \mathrm{C}(2)-\mathrm{Fe}(1)-\mathrm{C}(10) & 127.69(10) \\ \mathrm{C}(6)-\mathrm{Fe}(1)-\mathrm{C}(10) & 40.77(13) \\ \mathrm{C}(5)-\mathrm{Fe}(1)-\mathrm{C}(10) & 117.63(12) \\ \mathrm{C}(2)-\mathrm{Fe}(1)-\mathrm{C}(1) & 40.90(9) \\ \mathrm{C}(6)-\mathrm{Fe}(1)-\mathrm{C}(1) & 118.05(10) \\ \mathrm{C}(5)-\mathrm{Fe}(1)-\mathrm{C}(1) & 41.30(9) \\ \mathrm{C}(10)-\mathrm{Fe}(1)-\mathrm{C}(1) & 107.21(10) \\ \mathrm{C}(2)-\mathrm{Fe}(1)-\mathrm{C}(7) & 119.14(11) \\ \mathrm{C}(6)-\mathrm{Fe}(1)-\mathrm{C}(7) & 40.32(13) \\ \mathrm{C}(5)-\mathrm{Fe}(1)-\mathrm{C}(7) & 165.70(11) \\ \mathrm{C}(10)-\mathrm{Fe}(1)-\mathrm{C}(7) & 67.96(12) \\ \mathrm{C}(1)-\mathrm{Fe}(1)-\mathrm{C}(7) & 152.34(11) \\ \mathrm{C}(2)-\mathrm{Fe}(1)-\mathrm{C}(8) & 153.03(11) \\ \mathrm{C}(6)-\mathrm{Fe}(1)-\mathrm{C}(8) & 68.20(11) \\ & \end{array}$




\begin{tabular}{|c|c|}
\hline $\mathrm{C}(5)-\mathrm{Fe}(1)-\mathrm{C}(8)$ & $127.15(11)$ \\
\hline $\mathrm{C}(10)-\mathrm{Fe}(1)-\mathrm{C}(8)$ & $67.97(11)$ \\
\hline $\mathrm{C}(1)-\mathrm{Fe}(1)-\mathrm{C}(8)$ & $165.13(10)$ \\
\hline $\mathrm{C}(7)-\mathrm{Fe}(1)-\mathrm{C}(8)$ & $40.56(11)$ \\
\hline $\mathrm{C}(2)-\mathrm{Fe}(1)-\mathrm{C}(4)$ & $68.40(10)$ \\
\hline $\mathrm{C}(6)-\mathrm{Fe}(1)-\mathrm{C}(4)$ & $166.01(13)$ \\
\hline $\mathrm{C}(5)-\mathrm{Fe}(1)-\mathrm{C}(4)$ & $40.88(11)$ \\
\hline $\mathrm{C}(10)-\mathrm{Fe}(1)-\mathrm{C}(4)$ & $152.00(12)$ \\
\hline $\mathrm{C}(1)-\mathrm{Fe}(1)-\mathrm{C}(4)$ & $68.79(10)$ \\
\hline $\mathrm{C}(7)-\mathrm{Fe}(1)-\mathrm{C}(4)$ & $128.46(12)$ \\
\hline $\mathrm{C}(8)-\mathrm{Fe}(1)-\mathrm{C}(4)$ & $108.42(11)$ \\
\hline $\mathrm{C}(2)-\mathrm{Fe}(1)-\mathrm{C}(3)$ & $40.48(10)$ \\
\hline $\mathrm{C}(6)-\mathrm{Fe}(1)-\mathrm{C}(3)$ & $128.03(12)$ \\
\hline $\mathrm{C}(5)-\mathrm{Fe}(1)-\mathrm{C}(3)$ & $68.86(11)$ \\
\hline $\mathrm{C}(10)-\mathrm{Fe}(1)-\mathrm{C}(3)$ & $165.79(11)$ \\
\hline $\mathrm{C}(1)-\mathrm{Fe}(1)-\mathrm{C}(3)$ & $68.62(9)$ \\
\hline $\mathrm{C}(7)-\mathrm{Fe}(1)-\mathrm{C}(3)$ & $109.00(11)$ \\
\hline $\mathrm{C}(8)-\mathrm{Fe}(1)-\mathrm{C}(3)$ & $119.46(10)$ \\
\hline $\mathrm{C}(4)-\mathrm{Fe}(1)-\mathrm{C}(3)$ & $40.67(11)$ \\
\hline $\mathrm{C}(2)-\mathrm{Fe}(1)-\mathrm{C}(9)$ & $165.26(10)$ \\
\hline $\mathrm{C}(6)-\mathrm{Fe}(1)-\mathrm{C}(9)$ & $68.47(11)$ \\
\hline $\mathrm{C}(5)-\mathrm{Fe}(1)-\mathrm{C}(9)$ & $106.86(11)$ \\
\hline $\mathrm{C}(10)-\mathrm{Fe}(1)-\mathrm{C}(9)$ & $40.50(11)$ \\
\hline $\mathrm{C}(1)-\mathrm{Fe}(1)-\mathrm{C}(9)$ & $126.90(10)$ \\
\hline $\mathrm{C}(7)-\mathrm{Fe}(1)-\mathrm{C}(9)$ & $68.17(11)$ \\
\hline $\mathrm{C}(8)-\mathrm{Fe}(1)-\mathrm{C}(9)$ & $40.46(11)$ \\
\hline $\mathrm{C}(4)-\mathrm{Fe}(1)-\mathrm{C}(9)$ & $118.44(11)$ \\
\hline $\mathrm{C}(3)-\mathrm{Fe}(1)-\mathrm{C}(9)$ & $152.73(10)$ \\
\hline $\mathrm{C}(18)-\mathrm{Fe}(2)-\mathrm{C}(20)$ & $69.10(10)$ \\
\hline $\mathrm{C}(18)-\mathrm{Fe}(2)-\mathrm{C}(13)$ & $107.29(11)$ \\
\hline $\mathrm{C}(20)-\mathrm{Fe}(2)-\mathrm{C}(13)$ & $166.30(11)$ \\
\hline $\mathrm{C}(18)-\mathrm{Fe}(2)-\mathrm{C}(12)$ & $127.09(11)$ \\
\hline $\mathrm{C}(20)-\mathrm{Fe}(2)-\mathrm{C}(12)$ & $152.02(11)$ \\
\hline $\mathrm{C}(13)-\mathrm{Fe}(2)-\mathrm{C}(12)$ & $40.53(11)$ \\
\hline $\mathrm{C}(18)-\mathrm{Fe}(2)-\mathrm{C}(11)$ & $164.91(10)$ \\
\hline $\mathrm{C}(20)-\mathrm{Fe}(2)-\mathrm{C}(11)$ & $118.71(11)$ \\
\hline $\mathrm{C}(13)-\mathrm{Fe}(2)-\mathrm{C}(11)$ & $68.17(12)$ \\
\hline
\end{tabular}




$\begin{array}{lc}\mathrm{C}(12)-\mathrm{Fe}(2)-\mathrm{C}(11) & 40.31(11) \\ \mathrm{C}(18)-\mathrm{Fe}(2)-\mathrm{C}(14) & 118.74(12) \\ \mathrm{C}(20)-\mathrm{Fe}(2)-\mathrm{C}(14) & 128.46(11) \\ \mathrm{C}(13)-\mathrm{Fe}(2)-\mathrm{C}(14) & 40.65(13) \\ \mathrm{C}(12)-\mathrm{Fe}(2)-\mathrm{C}(14) & 67.93(11) \\ \mathrm{C}(11)-\mathrm{Fe}(2)-\mathrm{C}(14) & 67.89(12) \\ \mathrm{C}(18)-\mathrm{Fe}(2)-\mathrm{C}(15) & 152.87(12) \\ \mathrm{C}(20)-\mathrm{Fe}(2)-\mathrm{C}(15) & 108.45(12) \\ \mathrm{C}(13)-\mathrm{Fe}(2)-\mathrm{C}(15) & 68.36(12) \\ \mathrm{C}(12)-\mathrm{Fe}(2)-\mathrm{C}(15) & 68.00(11) \\ \mathrm{C}(11)-\mathrm{Fe}(2)-\mathrm{C}(15) & 40.47(12) \\ \mathrm{C}(14)-\mathrm{Fe}(2)-\mathrm{C}(15) & 40.42(13) \\ \mathrm{C}(18)-\mathrm{Fe}(2)-\mathrm{C}(17) & 40.96(10) \\ \mathrm{C}(20)-\mathrm{Fe}(2)-\mathrm{C}(17) & 68.32(10) \\ \mathrm{C}(13)-\mathrm{Fe}(2)-\mathrm{C}(17) & 118.22(12) \\ \mathrm{C}(12)-\mathrm{Fe}(2)-\mathrm{C}(17) & 107.65(11) \\ \mathrm{C}(11)-\mathrm{Fe}(2)-\mathrm{C}(17) & 127.23(11) \\ \mathrm{C}(14)-\mathrm{Fe}(2)-\mathrm{C}(17) & 152.67(13) \\ \mathrm{C}(15)-\mathrm{Fe}(2)-\mathrm{C}(17) & 165.21(12) \\ \mathrm{C}(18)-\mathrm{Fe}(2)-\mathrm{C}(19) & 41.22(9) \\ \mathrm{C}(20)-\mathrm{Fe}(2)-\mathrm{C}(19) & 41.15(10) \\ \mathrm{C}(13)-\mathrm{Fe}(2)-\mathrm{C}(19) & 127.62(11) \\ \mathrm{C}(12)-\mathrm{Fe}(2)-\mathrm{C}(19) & 165.52(10) \\ \mathrm{C}(11)-\mathrm{Fe}(2)-\mathrm{C}(19) & 152.80(10) \\ \mathrm{C}(14)-\mathrm{Fe}(2)-\mathrm{C}(19) & 108.33(10) \\ \mathrm{C}(15)-\mathrm{Fe}(2)-\mathrm{C}(19) & 118.93(11) \\ \mathrm{C}(17)-\mathrm{Fe}(2)-\mathrm{C}(19) & 68.85(9) \\ \mathrm{C}(18)-\mathrm{Fe}(2)-\mathrm{C}(16) & 68.70(10) \\ \mathrm{C}(20)-\mathrm{Fe}(2)-\mathrm{C}(16) & 40.51(10) \\ \mathrm{C}(13)-\mathrm{Fe}(2)-\mathrm{C}(16) & 151.84(11) \\ \mathrm{C}(12)-\mathrm{Fe}(2)-\mathrm{C}(16) & 118.34(10) \\ \mathrm{C}(11)-\mathrm{Fe}(2)-\mathrm{C}(16) & 108.07(11) \\ \mathrm{C}(14)-\mathrm{Fe}(2)-\mathrm{C}(16) & 166.03(13) \\ \mathrm{C}(15)-\mathrm{Fe}(2)-\mathrm{C}(16) & 127.97(12) \\ \mathrm{C}(17)-\mathrm{Fe}(2)-\mathrm{C}(16) & 40.37(11) \\ \mathrm{C}(19)-\mathrm{Fe}(2)-\mathrm{C}(16) & 68.74(9) \\ \mathrm{C}(1)+\mathrm{P}(3)-\mathrm{C}(1) & 101.31(10) \\ \end{array}$




$\begin{array}{ll}\mathrm{C}(1) \# 1-\mathrm{P}(3)-\mathrm{C}(1) \# 2 & 101.31(10) \\ \mathrm{C}(1)-\mathrm{P}(3)-\mathrm{C}(1) \# 2 & 101.31(10) \\ \mathrm{C}(19) \# 3-\mathrm{P}(4)-\mathrm{C}(19) & 102.44(10) \\ \mathrm{C}(19) \# 3-\mathrm{P}(4)-\mathrm{C}(19) \# 4 & 102.44(10) \\ \mathrm{C}(19)-\mathrm{P}(4)-\mathrm{C}(19) \# 4 & 102.44(10)\end{array}$

Symmetry transformations used to generate equivalent atoms:

$\# 1-x+y+1,-x+1, z \quad \# 2-y+1, x-y, z \quad \# 3-x+y,-x+1, z$

$\# 4-\mathrm{y}+1, \mathrm{x}-\mathrm{y}+1, \mathrm{z}$ 
Table S4. Anisotropic displacement parameters $\left(\AA^{2} \times 10^{3}\right)$. The anisotropic displacement factor exponent takes the form: $-2 \pi^{2}\left[h^{2} a^{* 2} U^{11}+\ldots+2 h k a^{*} b^{*} U^{12}\right]$

\begin{tabular}{|c|c|c|c|c|c|c|}
\hline & U11 & $\mathrm{U}^{22}$ & $\mathrm{U}^{33}$ & $\mathrm{U}^{23}$ & $\mathrm{U}^{13}$ & U12 \\
\hline $\mathrm{C}(1)$ & $13(1)$ & $12(1)$ & $17(1)$ & $0(1)$ & $2(1)$ & $7(1)$ \\
\hline$C(2)$ & $18(1)$ & $14(1)$ & $16(1)$ & $0(1)$ & $-2(1)$ & $9(1)$ \\
\hline $\mathrm{C}(3)$ & $21(1)$ & $19(1)$ & $21(1)$ & $-6(1)$ & $-4(1)$ & $11(1)$ \\
\hline$C(4)$ & $15(1)$ & $13(1)$ & $31(1)$ & $-1(1)$ & $1(1)$ & $5(1)$ \\
\hline $\mathrm{C}(5)$ & $14(1)$ & $12(1)$ & $23(1)$ & $3(1)$ & $6(1)$ & $6(1)$ \\
\hline$C(6)$ & $22(1)$ & $29(1)$ & $40(2)$ & $13(1)$ & $12(1)$ & $19(1)$ \\
\hline$C(7)$ & $40(2)$ & $39(2)$ & $22(1)$ & $5(1)$ & $8(1)$ & $32(1)$ \\
\hline $\mathrm{C}(8)$ & $31(1)$ & $19(1)$ & $28(1)$ & $3(1)$ & $0(1)$ & $17(1)$ \\
\hline $\mathrm{C}(9)$ & $25(1)$ & $24(1)$ & $23(1)$ & $2(1)$ & $-1(1)$ & $16(1)$ \\
\hline$C(10)$ & $20(1)$ & $21(1)$ & $40(2)$ & $1(1)$ & $-6(1)$ & $13(1)$ \\
\hline $\mathrm{C}(11)$ & $25(1)$ & $26(1)$ & $27(2)$ & $2(1)$ & $-4(1)$ & $18(1)$ \\
\hline$C(12)$ & $29(1)$ & $21(1)$ & $30(1)$ & $-2(1)$ & $-1(1)$ & $17(1)$ \\
\hline$C(13)$ & $40(2)$ & $36(2)$ & $24(1)$ & $-2(1)$ & $5(1)$ & $29(1)$ \\
\hline$C(14)$ & $26(1)$ & $26(1)$ & $42(2)$ & $8(1)$ & $16(1)$ & $17(1)$ \\
\hline$C(15)$ & $17(1)$ & $26(1)$ & $41(2)$ & $-6(1)$ & $-3(1)$ & $13(1)$ \\
\hline$C(16)$ & $20(1)$ & $20(1)$ & $21(1)$ & $7(1)$ & $6(1)$ & 11(1) \\
\hline$C(17)$ & $20(1)$ & $14(1)$ & $28(1)$ & $2(1)$ & $0(1)$ & $8(1)$ \\
\hline$C(18)$ & $19(1)$ & $14(1)$ & $18(1)$ & $0(1)$ & $-1(1)$ & $9(1)$ \\
\hline$C(19)$ & $15(1)$ & $16(1)$ & $16(1)$ & $1(1)$ & $0(1)$ & $9(1)$ \\
\hline$C(20)$ & $20(1)$ & $19(1)$ & $17(1)$ & $-1(1)$ & $0(1)$ & $12(1)$ \\
\hline $\mathrm{C}(21)$ & $33(1)$ & $22(1)$ & $20(1)$ & $-5(1)$ & $-11(1)$ & $13(1)$ \\
\hline$C(22)$ & $27(1)$ & $21(1)$ & $24(1)$ & $6(1)$ & $11(1)$ & $12(1)$ \\
\hline $\mathrm{Fe}(1)$ & $17(1)$ & $13(1)$ & $17(1)$ & $1(1)$ & $1(1)$ & $9(1)$ \\
\hline $\mathrm{Fe}(2)$ & $18(1)$ & $15(1)$ & $16(1)$ & $1(1)$ & $0(1)$ & $9(1)$ \\
\hline $\mathrm{P}(3)$ & $12(1)$ & $12(1)$ & $12(1)$ & 0 & 0 & $6(1)$ \\
\hline $\mathrm{P}(4)$ & $14(1)$ & $14(1)$ & $13(1)$ & 0 & 0 & $7(1)$ \\
\hline
\end{tabular}


Table 5. Hydrogen coordinates ( x 10 $)$ and isotropic displacement parameters $\left(\AA^{2} \times 10^{3}\right)$.

\begin{tabular}{|c|c|c|c|c|}
\hline & $\mathrm{x}$ & $\mathrm{y}$ & $\mathrm{z}$ & $\mathrm{U}(\mathrm{eq})$ \\
\hline $\mathrm{H}(2)$ & $6960(17)$ & $4174(17)$ & $1420(20)$ & $13(6)$ \\
\hline $\mathrm{H}(3)$ & $7940(20)$ & $5620(20)$ & $1680(30)$ & $31(9)$ \\
\hline $\mathrm{H}(4)$ & $8503(19)$ & $6320(20)$ & $-20(20)$ & $22(7)$ \\
\hline $\mathrm{H}(6)$ & $5340(20)$ & $4290(20)$ & $1010(30)$ & $37(9)$ \\
\hline $\mathrm{H}(7)$ & $6210(20)$ & $5740(20)$ & $1570(30)$ & $38(9)$ \\
\hline $\mathrm{H}(8)$ & 6934(19) & $6640(20)$ & $30(30)$ & $22(8)$ \\
\hline $\mathrm{H}(9)$ & $6484(17)$ & $5754(16)$ & $-1570(20)$ & $12(6)$ \\
\hline $\mathrm{H}(10)$ & $5466(17)$ & $4272(18)$ & $-940(20)$ & $17(7)$ \\
\hline $\mathrm{H}(11)$ & $3660(20)$ & $4100(20)$ & $-190(30)$ & $34(9)$ \\
\hline $\mathrm{H}(12)$ & $2920(20)$ & $3250(20)$ & $-1770(30)$ & $28(8)$ \\
\hline $\mathrm{H}(13)$ & $3460(20)$ & $4240(20)$ & $-3270(30)$ & $40(10)$ \\
\hline $\mathrm{H}(14)$ & $4510(20)$ & $5630(20)$ & $-2620(30)$ & $41(10)$ \\
\hline $\mathrm{H}(15)$ & $4620(20)$ & $5550(20)$ & $-720(30)$ & $34(9)$ \\
\hline $\mathrm{H}(16)$ & $1980(20)$ & $4240(20)$ & $-40(30)$ & $31(9)$ \\
\hline $\mathrm{H}(17)$ & $1440(20)$ & $3700(20)$ & $-1790(30)$ & $30(8)$ \\
\hline $\mathrm{H}(20)$ & $3007(18)$ & 5794(19) & $-140(20)$ & $21(7)$ \\
\hline $\mathrm{H}(21 \mathrm{~A})$ & $1710(20)$ & $5000(20)$ & $-3750(30)$ & $45(10)$ \\
\hline $\mathrm{H}(21 \mathrm{~B})$ & $2630(20)$ & $5180(20)$ & $-3850(30)$ & $29(8)$ \\
\hline $\mathrm{H}(21 \mathrm{C})$ & 1990(20) & $4330(30)$ & $-3600(30)$ & $44(10)$ \\
\hline $\mathrm{H}(22 \mathrm{~A})$ & $8010(30)$ & $5770(30)$ & $-1960(30)$ & $52(11)$ \\
\hline $\mathrm{H}(22 \mathrm{~B})$ & $7430(20)$ & $4900(20)$ & $-2180(30)$ & $33(9)$ \\
\hline $\mathrm{H}(22 \mathrm{C})$ & $8300(30)$ & $5140(20)$ & $-2020(30)$ & $44(10)$ \\
\hline
\end{tabular}


${ }^{1}$ H NMR of 2-methyl-1-diphenylphosphinoferrocene 11.

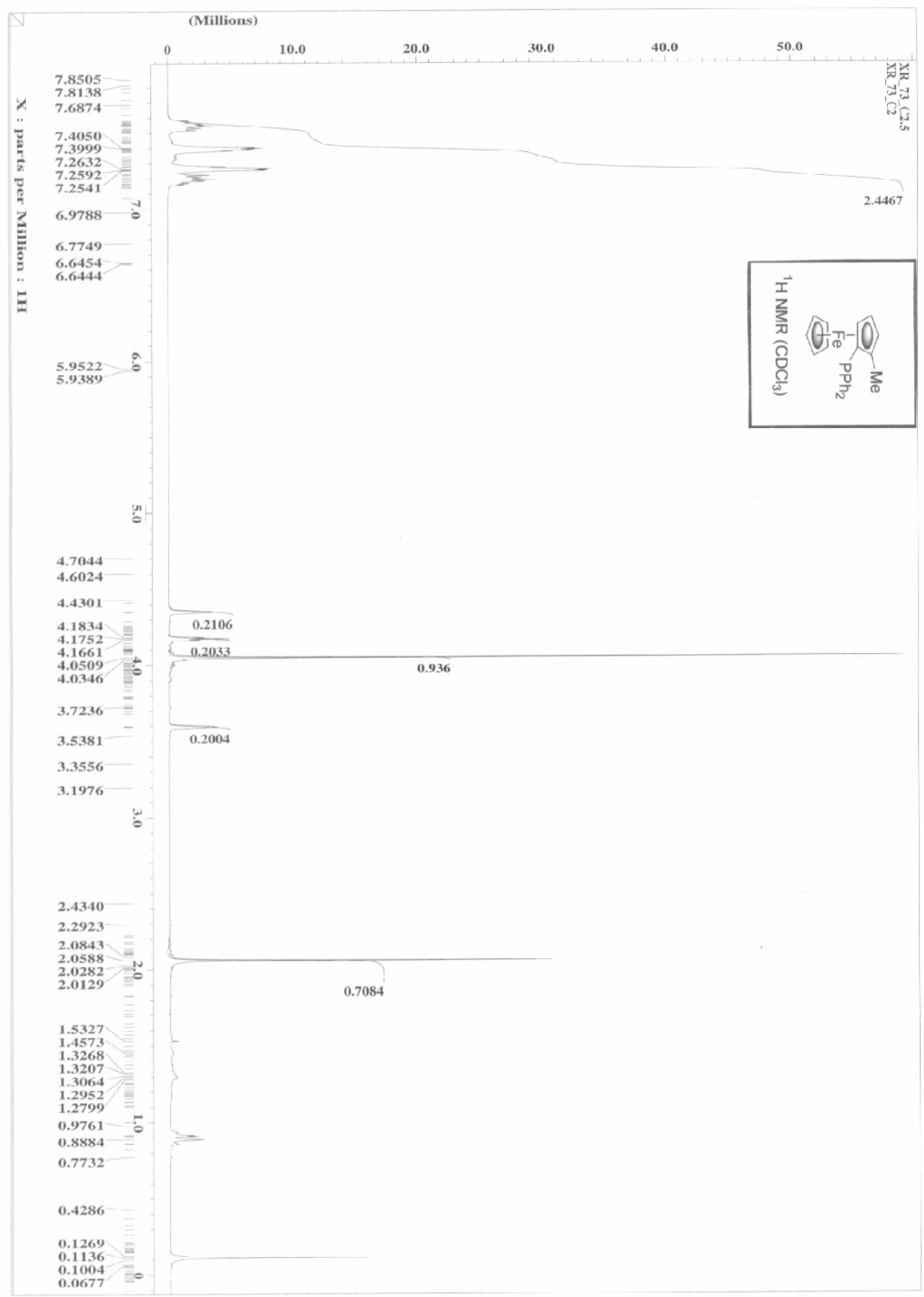


${ }^{31}$ P NMR of 2-methyl-1-diphenylphosphinoferrocene 11.

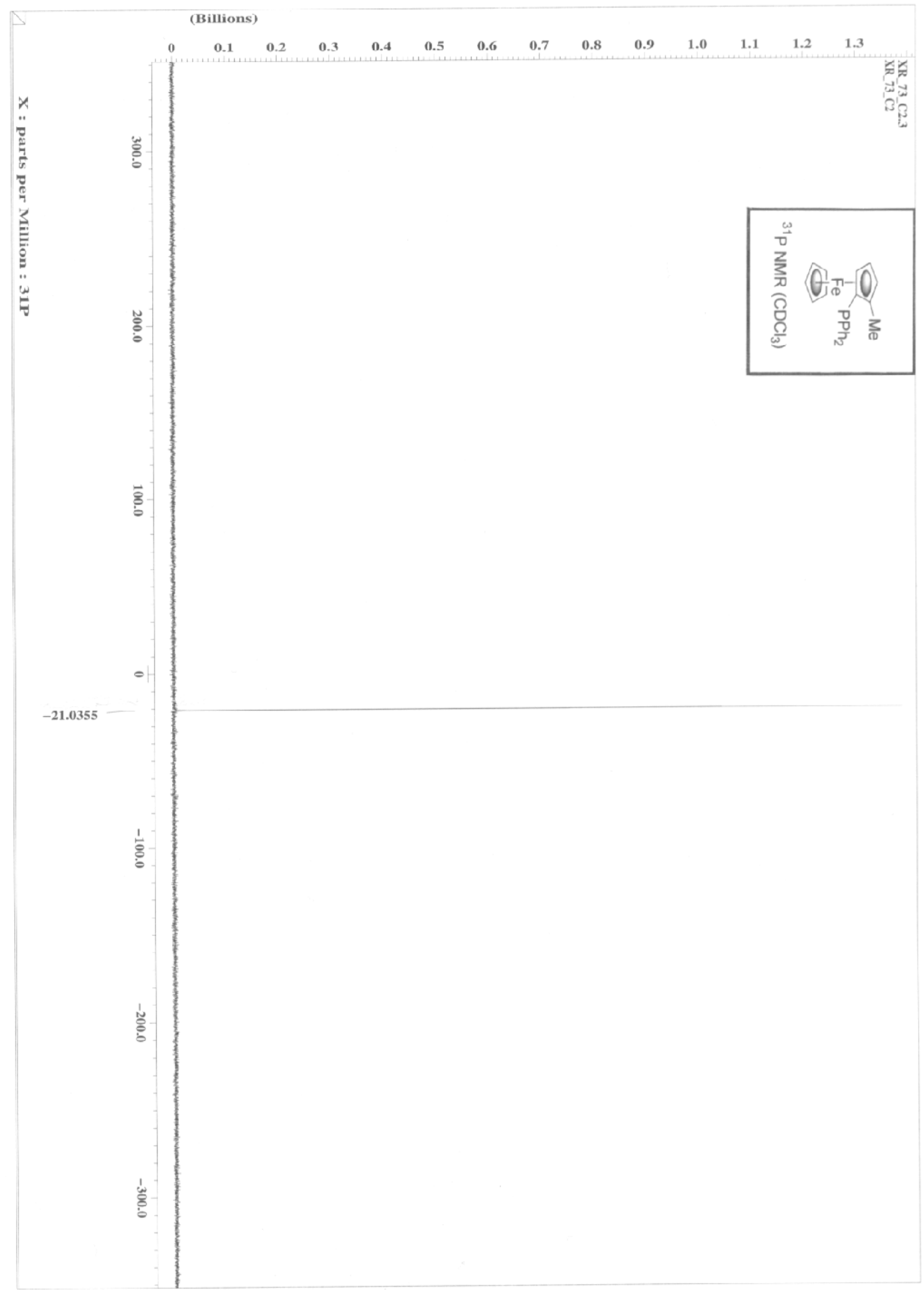


${ }^{1} \mathrm{H}$ NMR of 2-methyl-1-dicyclohexylphosphinoferrocene 12.

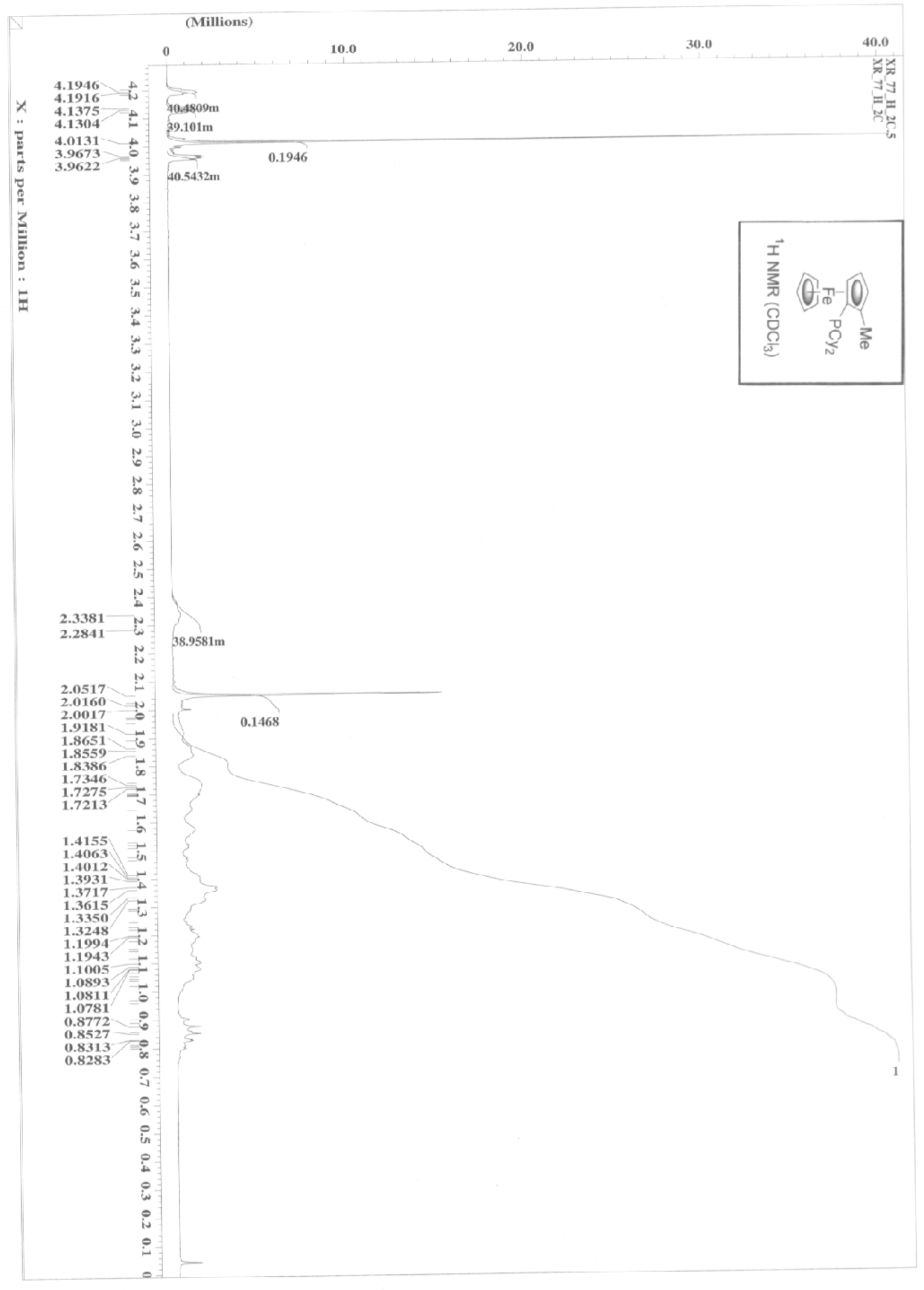


${ }^{31} \mathrm{P}$ NMR of 2-methyl-1-dicyclohexylphosphinoferrocene12.

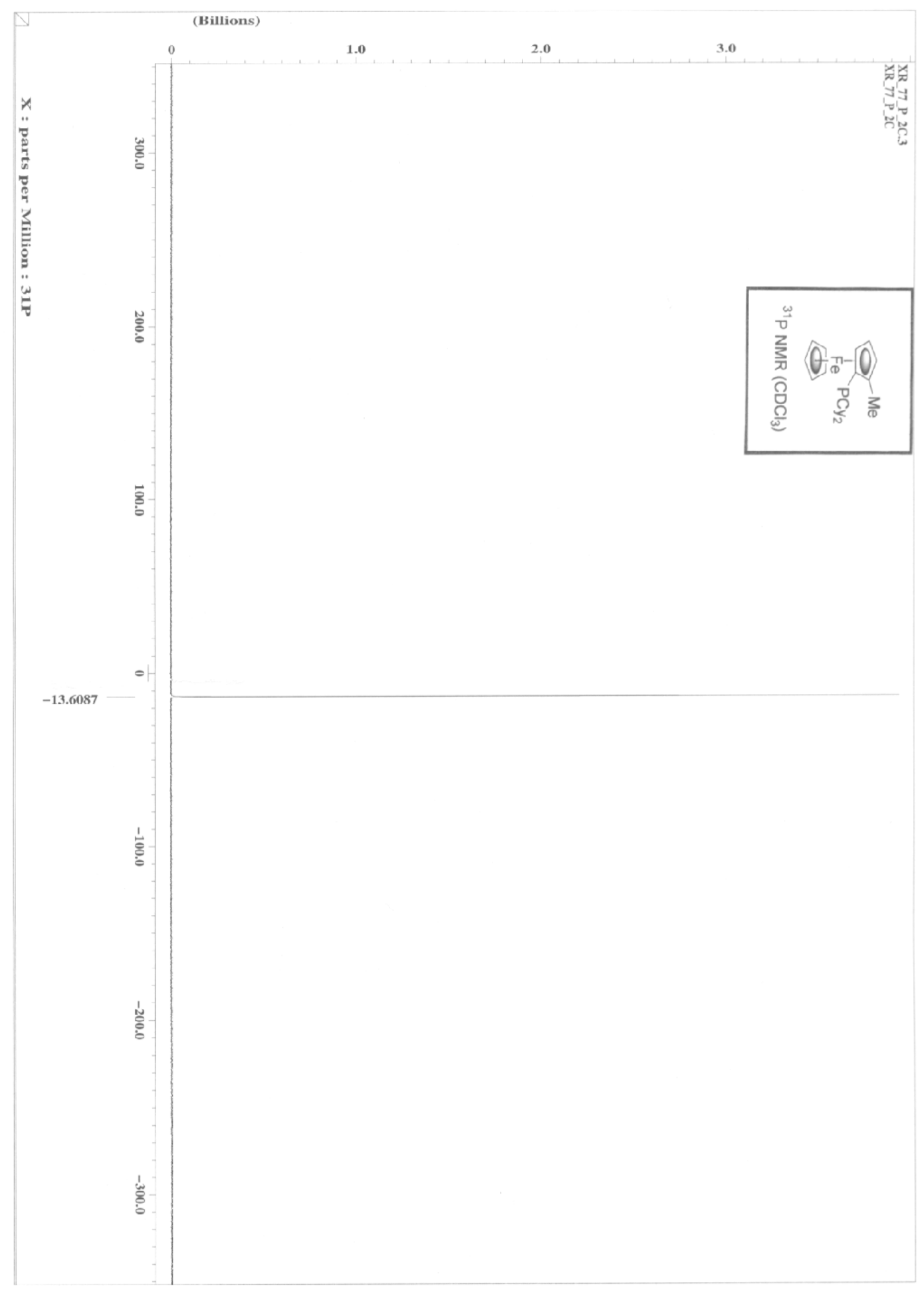


${ }^{1} \mathrm{H}$ NMR of 2-methyl-1-di-tert-butylphosphinoferrocene 13.

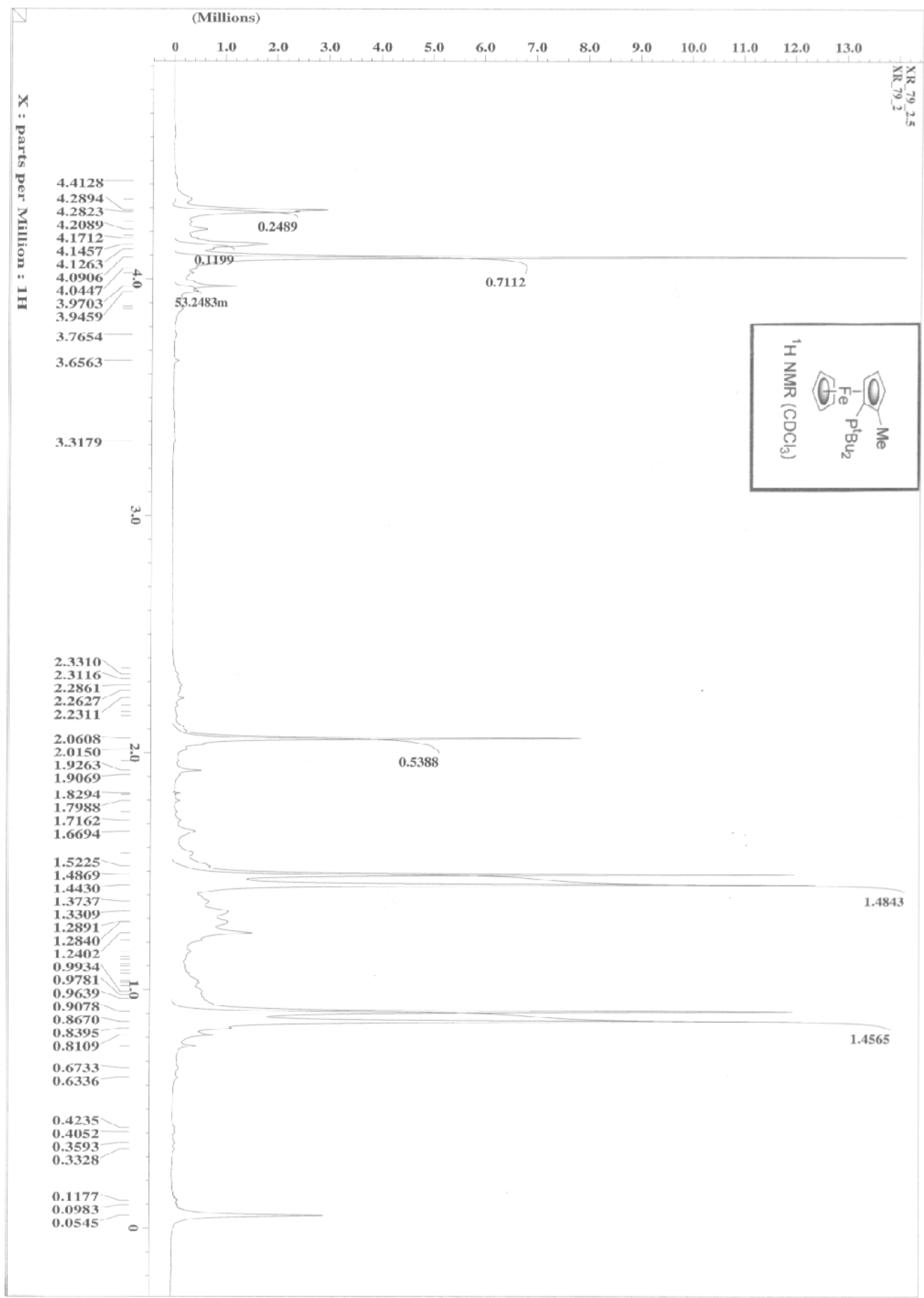


${ }^{31} \mathrm{P}$ NMR of 2-methyl-1-di-tert-butylphosphinoferrocene 13.

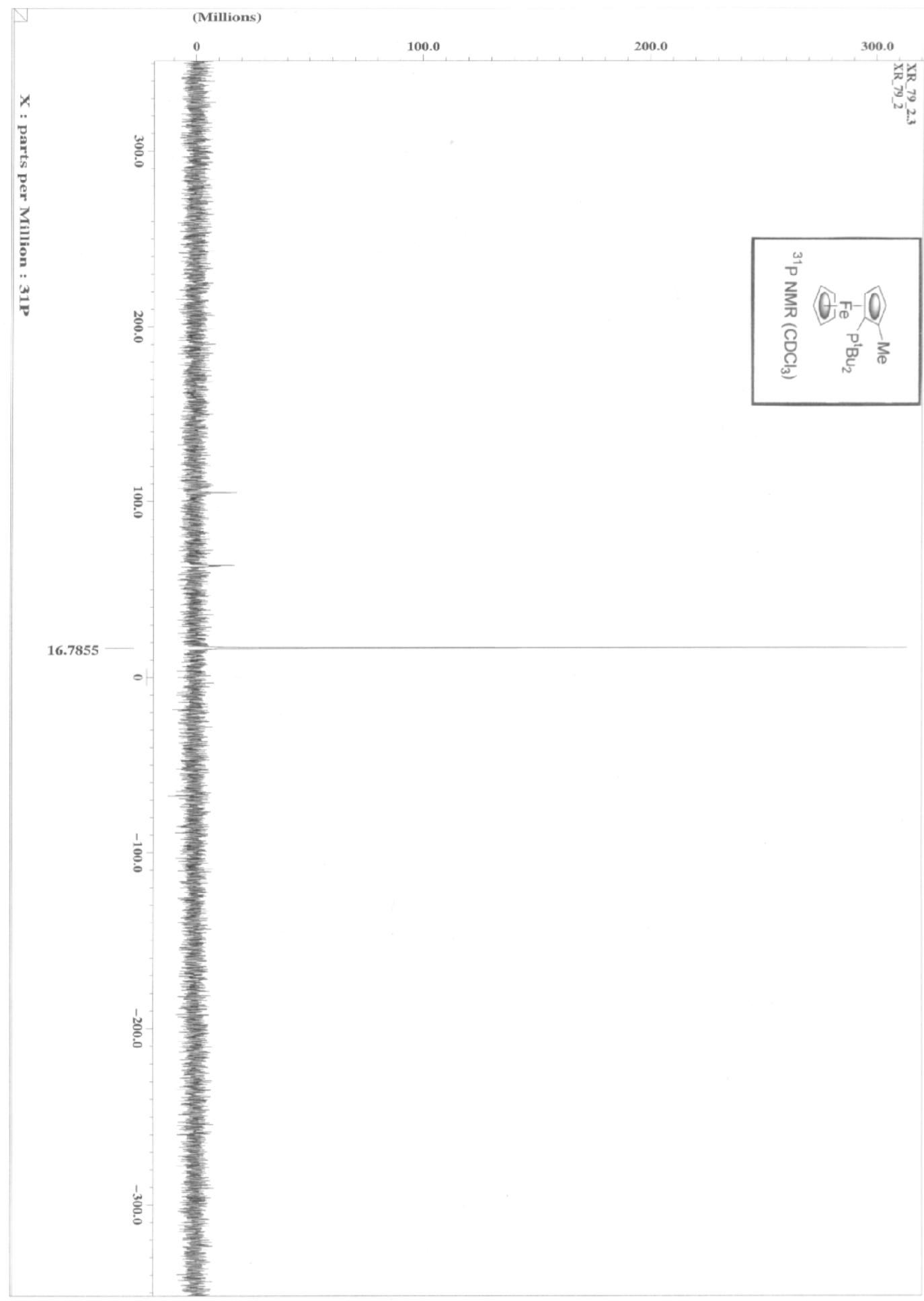

